\title{
Lagrangian-Eulerian approximation methods for balance laws and hyperbolic conservation laws
}

\section{Métodos de aproximación Lagrangiano-Eulerianos para leyes de equilibrio y leyes de conservación hiperbólicas}

\author{
Eduardo Abreu ${ }^{1}$, John Pérez ${ }^{2}$, Arthur Santo ${ }^{3}$ \\ ${ }^{1}$ Institute of Mathematics, Statistics and Scientific Computing - UNICAMP, Campinas, Brasil. Email: eabreu@ime.unicamp.br \\ ${ }^{2}$ Facultad de Ciencias Exactas y Aplicadas, ITM Institución Universitaria, Medellín, Colombia. Email: jhonperez@itm.edu.co \\ ${ }^{3}$ Institute of Mathematics, Statistics and Scientific Computing - UNICAMP, Campinas, Brasil. Email: arthurm@ime.unicamp.br
}

RECEIVED: February 28, 2017. ACCEPTED: May 27, 2017. FINAL VERSION: October 25, 2017.

\section{RESUMEN}

Un nuevo volumen finito de control es presentado en un enfoque Lagrangiano-Euleriano (ver artículos [1, 28]), en este, un dominio de espacio-tiempo es estudiado con el fin de diseñar un esquema localmente conservativo. Tal esquema tiene en cuenta el delicado balance no linear, entre las aproximaciones numéricas del flujo hiperbólico y el término fuente, en problemas de ley de balance ligados con leyes de conservación puramente hiperbólicas. Además, combinando algunas ideas de este nuevo enfoque, hacemos una construcción formal de un nuevo algoritmo para resolver importantes problemas de leyes de conservación en dos dimensiones espaciales. Un conjunto pertinente de experimentos numéricos para diferentes modelos es presentado para mostrar evidencia que soluciones cualitativamente correctas son aproximadas.

PALABRAS CLAVE: Leyes de conservación; Lagrangiano-Euleriano; volumen finito.

\begin{abstract}
A new finite control volume in a Lagrangian-Eulerian framework is presented (see papers $[1,28]$ ), in which a local space-time domain is studied, in order to design a locally conservative scheme. Such scheme accounts for the delicate nonlinear balance between the numerical approximations of the hyperbolic flux and the source term for balance law problems linked to the purely hyperbolic character of conservation laws. Furthermore, by combining the ideas of this new approach, we give a formal construction of a new algorithm for solving several nonlinear hyperbolic conservation laws in two space dimensions. Here, a set of pertinent numerical experiments for distinct models is presented to evidence that we are calculating the correct qualitatively good solutions.
\end{abstract}

KEYWORDS: Conservation laws; Lagrangian-Eulerian; finite volume.

\section{INTRODUCTION}

In this work we explore a locally conservative and spacetime finite control volume in a Lagrangian-Eulerian framework (see $[13,14,15,26]$ ), first developed in the context of purely hyperbolic conservation laws, in order to design a locally conservative scheme to account the balance between numerical approximations of the hyperbolic flux function and the source term linked to steady solutions. In [1,28], such Lagrangian framework was extended to design a locally conservative Lagrangian-Eulerian scheme to account the balance between numerical approximations of the hyperbolic flux function and the source term linked to steady-state

Este artículo puede compartirse bajo la licencia CC BY-ND 4.0 y se referencia usando el siguiente formato: E. Abreu, J. Pérez, A. Santo, "Lagrangian-Eulerian approximation methods for balance laws and hyperbolic conservation laws," Rev. UIS Ing., vol. 17, no. 1, pp. 191-200, 2018. Doi: https://doi.org/10.18273/revuin.v17n1-2018018 
solutions for one-dimensional nonlinear balance law problems - scalar and system - given by:

$$
\begin{aligned}
& \frac{\partial u(x, t)}{\partial t}+\frac{\partial f(u(x, t))}{\partial x}=G(u), \\
& u(x, 0)=\varphi(x), \\
& -\infty<x<\infty, \quad t>0,
\end{aligned}
$$

We made use of the innovative ideas of $[13,14,15,26]$ to develop a locally conservative by construction Lagrangian-Eulerian approach as an alternative way to define accurate approximation for purely advection (hyperbolic) problems in two dimensions, virtually free of numerical diffusion $[2,14,20]$. The new LagrangianEulerian scheme is aimed to be independent of any particular structure of the source term. The designed scheme makes no use of Riemann solvers for the resolution of (local) Riemann problems, but, if Riemann solutions are available for a particular problem it is somewhat natural to incorporate such information into the procedure. This yields flexibility to the development of distinct numerical strategies upon the specific model under consideration.

We are interested in numerically solving balance law problems linked to the homogeneous purely hyperbolic conservation law counterpart. In this work, we aim to present a Lagrangian-Eulerian scheme in a cell-centered framework devoted to this task. This scheme consists on another attempt to deal with the difficult issue of the wellbalancing between the computation of the numerical flux function and the source term by means of a natural unbiased upwinding approach, which in turn is Riemann solver-free and seems to be able to handle nonlinear scalar and system problems. A variety of efficient numerical schemes for hyperbolic systems of conservation laws has been developed in the recent past for different problem settings. These schemes evolved following the natural understanding of fundamental concepts from the theory of nonlinear hyperbolic conservation laws concerning the characteristic surfaces properties, existence, uniqueness, and solution of the Riemann problem; see, e.g, [4, 7, 10, 22, 24, 25, 32].

In addition, for a scalar balance law, the solution strongly depends on certain properties of the source term (see [16, $10,22,4,7,24,25])$. For example, when the source term is a non-increasing function, the total variation of the exact solution of the scalar balance law is also a nonincreasing function, as in the homogeneous case (see, e.g., $[16,10])$. In general, however, the source term might not be decreasing (see $[6,11,27]$ ) and some semiimplicit and fully implicit scheme are not applicable, at least in a straightforward manner $[6,11]$.
In $[1,2,13,14,15,20,30]$ the authors present distinct Lagrangian-Eulerian formulations to the linear case [3, 20] and non-linear $[1,2,12,14,30]$ transport flow problems; to the purely linear transport problem the space-time integral curves coincide with characteristic equations [2, 20] (see also [4]). Such LagrangianEulerian approach provides a very accurate solution to purely advective problems, virtually free of numerical diffusion. Such schemes are derived from the divergence forms of the equations. It is the use of the divergence form of a parabolic equation that allows relatively easy localization of desired conservation principles in a form amenable to the application of finite element or finite volume approaches in a locally conservative fashion. Essentially, this formulation evolves [2, 12, 14] (see also $[2,20,30]$ and references therein) from the efforts to develop fast, accurate, and stable versions of Modified Method of Characteristics numerical methods for transport-dominated diffusive systems, with the primary objective of the evolution being the incorporation of changes in these procedures to obtain the preservation of desired conservation principles. Here we want to follow such ideas to the case of balance laws.

In section 2 we briefly show the ideas of the construction of the method for scalar problems of hyperbolic conservation laws and balance laws. In section 3 it is shown, also briefly, the extension of method to numerically solve hyperbolic conservation laws in two spatial dimensions. Finally, we show some numerical experiments in section 4 .

\section{THE LAGRANGIAN-EULERIAN METHOD}

As in $[1,28]$, we consider the general balance law

$$
\begin{aligned}
& \frac{\partial u(x, t)}{\partial t}+\frac{\partial f(u(x, t))}{\partial x}=G(u), \\
& u(x, 0)=\varphi(x), \\
& -\infty<x<\infty, \quad t>0,
\end{aligned}
$$

where $G(u)$ is a integrable function.

Following $[1,2,14,28,20]$, we consider the finitevolume cell centers $D_{j}=\left\{(t, x): t^{n} \leq t \leq\right.$ $\left.t^{\{n+1\}}, \sigma_{j}(t) \leq x \leq \sigma_{j+1}(t)\right\}$ where $\sigma_{j}(t)$ is a parameterized curve such that $\sigma_{j}(t)$ is a solution of the system of ordinary differential equations $\frac{d \sigma_{j}(t)}{d t}=\frac{f\left(U_{j}^{n}\right)}{U_{j}^{n}}$ with initial condition $\sigma_{j}\left(t^{n}\right)=x_{j}^{n}$. Also, we get a CFL condition of the form $\lambda\left|f_{j}^{n}\right| \leq \frac{\sqrt{2}}{2}$, where $\lambda=\frac{k}{h}$ and $f_{j}^{n}=\frac{f\left(U_{j}^{n}\right)}{U_{j}^{n}}$. 
Next, from the divergence theorem $[2,14,28]$ reads:

$$
\begin{gathered}
\iint_{D_{j}} \nabla_{t, x}\left[\begin{array}{c}
u \\
f(u)
\end{array}\right] d V=\iint_{D_{j}} G(u) d V \Leftrightarrow \\
\oint_{\partial D_{j}}\left[\begin{array}{c}
u \\
f(u)
\end{array}\right] \cdot n d s=\iint_{D_{j}} G(u) d A
\end{gathered}
$$

Imposition of impermeability condition over curves $\sigma_{j}(t)$ in boundary $\partial D_{j}$, equation (3), implies that the integrals over curves $\sigma_{j}(t)$ vanish. From this fact, the region $D_{j}$ will be called "Integral tube" as a natural consequence of (3).

The previous item allows us to define the evolution step from time $t^{n}$ to $t^{n+1}$ as

$$
\begin{gathered}
\bar{U}_{j}^{n+1}=\frac{1}{h_{j}^{n+1}}\left[\int_{x_{j}^{n}}^{x_{j+1}^{n}} u\left(x, t^{n}\right) d x+\iint_{D_{j}} G(u) d A\right], \\
\text { where } h_{j}^{n+1}=h+\left(f_{j+1}^{n}-f_{j}^{n}\right) k, k=\Delta t^{n} .
\end{gathered}
$$

Next, the local approximation $\bar{U}_{j}^{n+1}, j \in Z$, is projected over the original grid, as follows:

$$
U_{j}^{n+1}=\frac{1}{h}\left(\left(\frac{h}{2}-f_{j}^{n} k^{n}\right) \bar{U}_{j-1}^{n+1}+\left(\frac{h}{2}+f_{j}^{n} k^{n}\right) \bar{U}_{j}^{n+1}\right) .
$$

In the linear case, along with $G(u)=0$, the approximations to the simple scalar conservation law

$$
\begin{aligned}
& \frac{\partial u(x, t)}{\partial t}+\frac{\partial(a u)}{\partial x}=0, \\
& u(x, 0)=\varphi(x), \\
& -\infty<x<\infty, \quad t>0,
\end{aligned}
$$

Is also the simple finite difference Lagrangian-Eulerian scheme $[1,28]$

$$
U_{j}^{n+1}=\frac{1}{4}\left[U_{j-1}^{n}+2 U_{j}^{n}+U_{j+1}^{n}\right]-\frac{a k}{h}\left[U_{j+1}^{n}-U_{j-1}^{n}\right]
$$

Based on numerical domain of influence and domain of dependence, for (7) the following Courant-FriedrichsLewy CFL like stability condition is $a k h^{-1}<2^{-1}$.

\section{THE NEW LAGRANGIAN- EULERIAN FRAMEWORK FOR TWO-DIMENSIONAL HYPERBOLIC CONSERVATION LAWS}

We discuss a Lagragian-Eulerian technique for approximation of the following two-dimensional initial value problem for conservation laws (see $[1,28]$ ),

$$
\begin{gathered}
\frac{\partial u}{\partial t}+\frac{\partial f(u)}{\partial x}+\frac{\partial g(u)}{\partial y}=0 \\
u\left(x, y, t_{0}\right)=\eta(x, y) \\
(x, y, t) \in \Omega \times\left(t_{0}, T\right]
\end{gathered}
$$

Along with the approximations of derivatives respectively. We write $(8)$ as, (for $w=(x, y, t))$

$$
\left\{\begin{array}{l}
\frac{\partial u}{\partial t}+\frac{\partial f(u)}{\partial x}=-\left(\frac{\partial g(u)}{\partial y}\right)_{j}, w \in D_{i, j}^{n} \\
\frac{\partial u}{\partial t}+\frac{\partial g(u)}{\partial x}=-\left(\frac{\partial f(u)}{\partial y}\right)_{i}, w \in D_{i, j}^{n+\frac{1}{2}}
\end{array}\right.
$$

As seen in the scalar case, we consider cell-centered finite-volume cell centers in the Lagrangian-framework as follows: $D_{i, j}^{n}=\left\{(x, y, t): t^{n} \leq t \leq t^{n+\frac{1}{2}}, y_{j-\frac{1}{2}}^{n} \leq y \leq\right.$ $\left.y_{j+\frac{1}{2}}^{n}, \sigma_{i}^{n}(t) \leq x \leq \sigma_{i+1}^{n}(t)\right\} \quad$ and $\quad D_{i, j}^{n+\frac{1}{2}}=$ $\left\{(x, y, t): t^{n} \leq t \leq t^{n+\frac{1}{2}}, x_{j-\frac{1}{2}}^{n} \leq x \leq x_{j+\frac{1}{2}}^{n} \gamma_{i}^{n+\frac{1}{2}}(t) \leq\right.$ $\left.y \leq \gamma_{i+1}^{n+\frac{1}{2}}(t)\right\}$, where $\sigma_{i}^{n}(t)$ and $\gamma_{i}^{n+\frac{1}{2}}(t)$ are solutions of $\frac{d \Theta(t)}{d t}=\frac{H(u)}{u} \approx \frac{H\left(U^{*}\right)}{U^{*}}, H=f, g$

We prove the stability in $l_{h}^{p}$ norm, under CFL condition $\lambda\left|f H_{j}^{n}\right| \leq \frac{\sqrt{2}}{2}$, where $\lambda=\frac{k}{h}$,

Finally, we sequentially solve each balance law problem given by (9)-(10), in each control volume and respectively, by using the numerical scheme (3), (4) and (5) as a building block (see also [28] for more details).

\section{NUMERICAL EXPERIMENTS}

We provide some numerical experiments with specific model test problems, in order to show the algorithm behavior and its applicability, linked to the firm mathematical underpinnings used into the previous sections for the construction of the proposed new algorithm.

Insight in the qualitative behavior (see numerical experiments from Figure 1 to Figure 4) can be obtained by regarding the so-called modified equations of the discretization. It is worth mentioning that here we will use modified equations with the aim of understanding the qualitative behavior of approximations (related to the two-level Lagrangian-Eulerian scheme (7)) in the spirit of critical work of [5] (see also [17, 31]), that related it with the Fourier-von Neumann method for the linear stability analysis of the linear initial-value problem (Lax stability in PDEs as before) of two-level linear difference schemes. Let $-(\mathrm{x} ; \mathrm{t})$ in $\mathrm{C} 1$, with all bounded derivatives with respect to $\mathrm{x}$ and $\mathrm{t}$, in the setting of modified equations and plug this into (7) along with the stability results pointed out in $[1,28]$. 
In the linear case, the complicated behaviors exhibited by the Fourier series sums would serve as a good testing ground for the application of numerical methods for diffusive/dispersive waves to rough data. Thus, let us discuss the application of the Lagrangian-Eulerian scheme (7) with three types of initial data:

a. $C^{\infty}$ smooth data (see Figure 1 and Figure 2),

b. a Lipschitz initial data (see Figure 3) and

c. piecewise discontinuous initial data (Figure 4).

For all cases we will also show the observed numerical convergence rates (see Tables 1, 2 and 3) along with comments on the captions and figures. In other words, the classical convergence (consistency + stability) property of a numerical scheme does not suffice to guarantee its suitability for providing good approximations to the controls that might be needed in applications since such nice properties may be lost under numerical discretization as the mesh size tends to zero due to the existence of high-frequency spurious solutions for which the group velocity vanishes not fast enough. We will not suggest any specialized remedies since we are primary interest in discuss the properties (we have found) in the most simple framework, which is conducive to analysis and verification by representative numerical tests.

Table 1. Errors between the numerical approximations $(U)$ and exact solutions $(u)$ in $l_{h}^{1}\left(l_{h}^{2}\right)$ for problem $u_{t}+u_{x}=0$ with smooth initial condition at time frame $T=2$ and CFL condition equal to $\frac{\sqrt{2}}{2}$.

\begin{tabular}{|l|c|}
\hline Cells & Smooth $\|u-U\|_{l_{h}^{1}}\left(\|u-U\|_{l_{h}^{2}}\right)$ \\
\hline 32 & $5.921 \times 10^{-1}\left(2.980 \times 10^{-1}\right)$ \\
\hline 64 & $2.082 \times 10^{-1}\left(1.146 \times 10^{-1}\right)$ \\
\hline 128 & $5.571 \times 10^{-2}\left(3.171 \times 10^{-2}\right)$ \\
\hline 256 & $1.399 \times 10^{-2}\left(8.006 \times 10^{-3}\right)$ \\
\hline$E(h)$ & $1.673 h^{1.811}\left(0.835 h^{1.75}\right)$ \\
\hline
\end{tabular}

Source: Author development.

Table 2. Errors between the numerical approximations $(U)$ and exact solutions $(u)$ in $l_{h}^{1}\left(l_{h}^{2}\right)$ for problem $u_{t}+u_{x}=0$ with continuous initial condition at time frame $T=2$ and CFL condition equal to $\frac{\sqrt{2}}{2}$.

\begin{tabular}{|l|c|}
\hline Cells & Continuous $\|u-U\|_{l_{h}^{1}}\left(\|u-U\|_{l_{h}^{2}}\right)$ \\
\hline 32 & $7.834 \times 10^{-1}\left(4.521 \times 10^{-1}\right)$ \\
\hline 64 & $3.898 \times 10^{-1}\left(2.267 \times 10^{-1}\right)$ \\
\hline 128 & $1.428 \times 10^{-1}\left(9.095 \times 10^{-2}\right)$ \\
\hline 256 & $6.541 \times 10^{-2}\left(4.869 \times 10^{-2}\right)$ \\
\hline$E(h)$ & $1.550 h^{1.219}\left(0.808 h^{1.096}\right)$ \\
\hline
\end{tabular}

Source: Author development.
Table 3. Errors between the numerical approximations $(U)$ and exact solutions $(u)$ in $l_{h}^{1}\left(l_{h}^{2}\right)$ for problem $u_{t}+u_{x}=0$ with non-continuous initial condition at time frame $T=2$ and CFL condition equal to $\frac{\sqrt{2}}{2}$.

\begin{tabular}{|l|c|}
\hline Cells & NonContin. $\|u-U\|_{l_{h}^{1}}\left(\|u-U\|_{l_{h}^{2}}\right)$ \\
\hline 32 & $1.192 \times 10^{-1}\left(5.454 \times 10^{-1}\right)$ \\
\hline 64 & $7.984 \times 10^{-1}\left(4.458 \times 10^{-1}\right)$ \\
\hline 128 & $5.639 \times 10^{-1}\left(4.374 \times 10^{-1}\right)$ \\
\hline 256 & $3.552 \times 10^{-1}\left(2.887 \times 10^{-1}\right)$ \\
\hline$E(h)$ & $1.679 h^{0.574}\left(0.646 h^{0.27}\right)$ \\
\hline
\end{tabular}

Source: Author development.

Approximate numerical solutions given by scheme (7) to the Linear hyperbolic PDE (6), are shown in Figure 1, along with smooth Gaussian initial condition $u(x, 0)=$ $\eta(x)=e^{-x^{2}}$. It is shown initial condition (top left) and computed solutions at $\mathrm{t}=2$ (top right), $\mathrm{t}=4$ (bottom left) and $\mathrm{t}=8$ (bottom right) with CFL number $v=\frac{\sqrt{2}}{2}$. As expected from the modified equation analysis the solutions start to exhibit dispersion since the range of the dominant dispersion regime is controlled by $\frac{1}{2}<v<\frac{\sqrt{2}}{2}$.

In $[1,28]$ we establish a convergence proof in $l^{2}$-space by means of von Neumann analysis for the LagrangianEulerian scheme, see Figure 2. Although the CFL number is in the range $\frac{1}{2}<v<\frac{\sqrt{2}}{2}$ where the diffusion is in balance or dominates the dispersion, the above numerical experiments related to those in Figure 1, illustrate the fact of entire truncation error vanishing (see right picture) at all grid points under grid refinement, as expected from previous theoretical analysis. At this point (left and right picture) notice the excellent resemblance between the exact and approximate solutions computed by the Lagrangian- Eulerian scheme with any reminiscence of the spurious effects from the numerical dispersion/diffusion artifacts.

The numerical experiment in Figure 3 illustrates again both cases where the diffusion are in balance (bottom left) or dominates (bottom middle) the dispersion as $h \rightarrow$ 0 with ratio $k=h$ fixed corresponding essentially to what is shown in Figure 1 and in Figure 2, but with a Lipschitz initial condition where $\eta(x)=x+1,-1<$ $x<0, \eta(x)=-x+1,0<x<1 \quad$ and $\eta(x)=0$ elsewhere. 

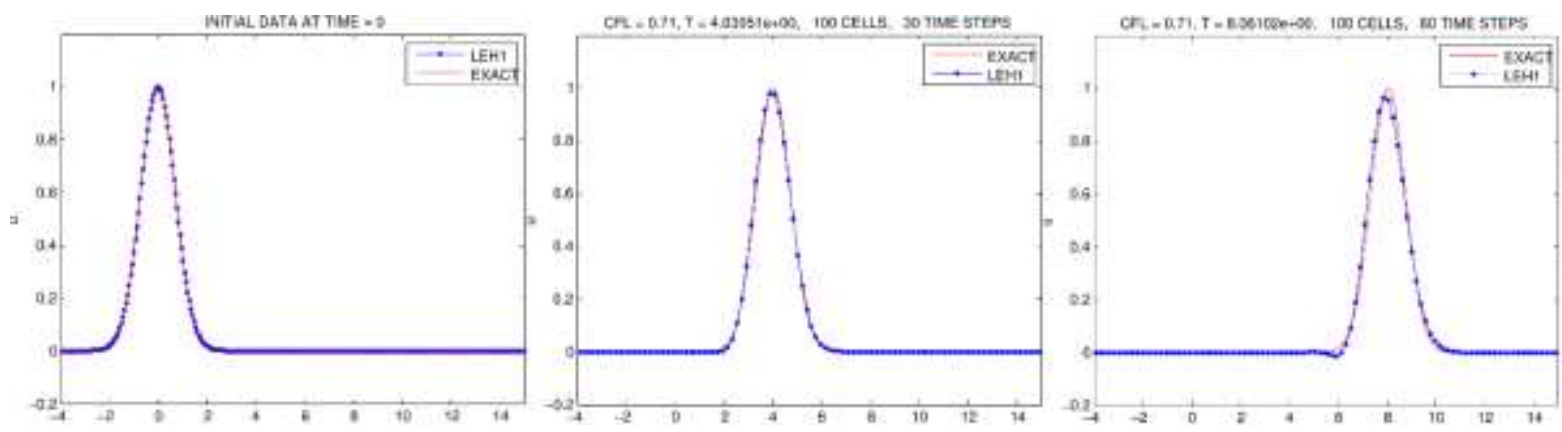

Figure 3. Numerical solutions given by scheme (7) along with smooth Gaussian initial condition.
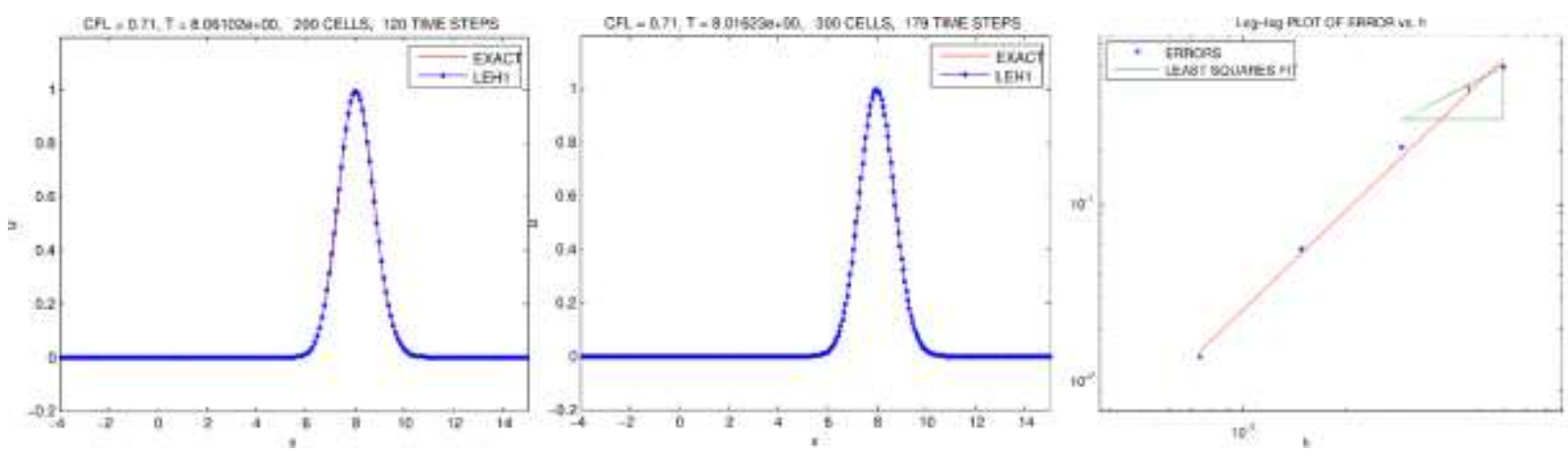

Figure 2. Convergence in $\mathrm{l}^{2}$-space.
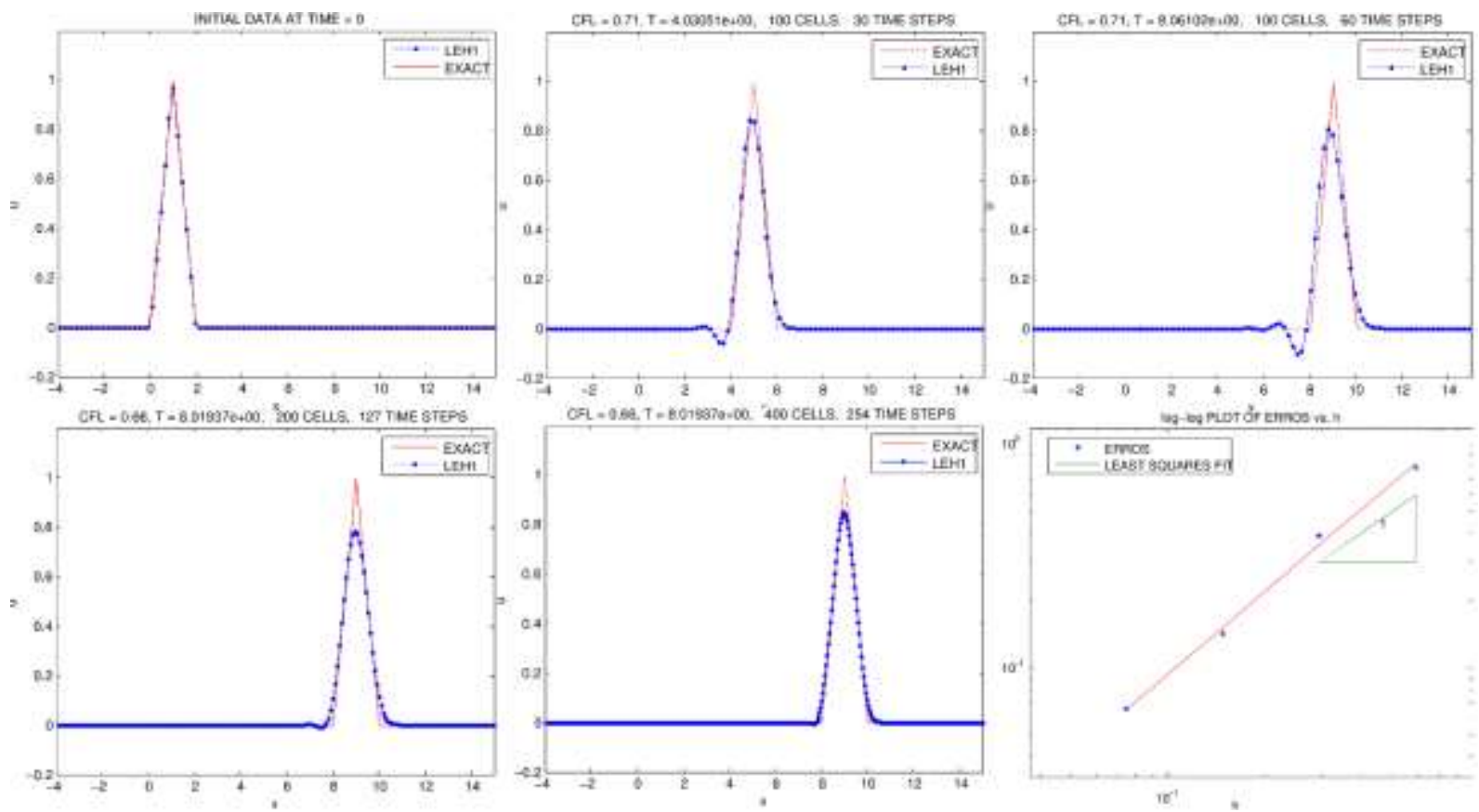

Figure 1. Numerical approximation with a Lipschitz initial condition.

Numerical experiment in Figure 4 illustrates again both cases where the diffusion are in balance (bottom left) or dominates (bottom middle) the dispersion as $h \rightarrow 0$ with ratio $k=h$ fixed corresponding essentially to what is shown in Figure 1 and in Figure 2, but with a discontinuous initial condition where $\eta(x)=1,-2<$ $x<2$ and $\eta(x)=0$ elsewhere. As in [23], in Figure 5, we show preliminary numerical approximations of the model problem with $f(u)=u$ and $G(u)=-\beta u(u-$ 1) $(u-\mu)$ and $\mu=0.5$, this model was used in [23] to 

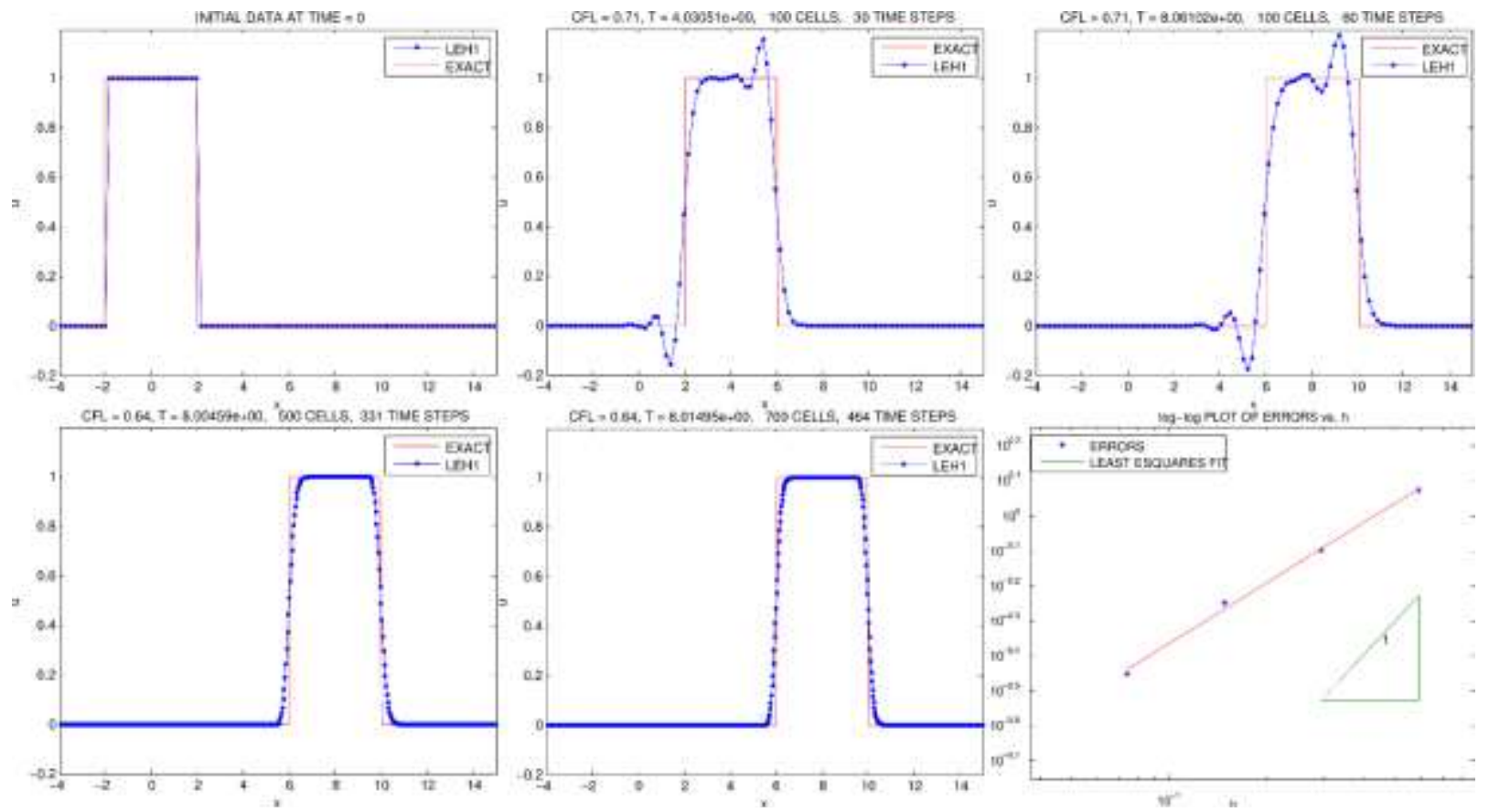

Figure 4. Numerical approximation with a discontinuous initial condition.
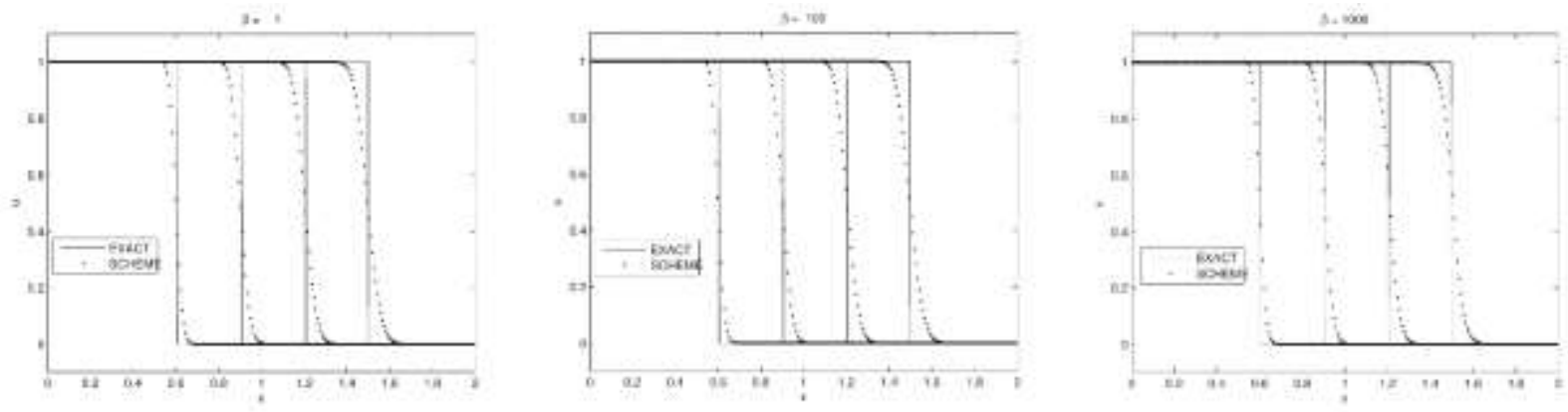

Figure 5. The model problem proposed by LeVeque and Yee in [23].

illustrate a current well-known deficiency of most numerical schemes for hyperbolic conservation laws with stiff source terms. These schemes obtain propagation at a nonphysical speed that is purely an artifact of the numerical method. The problem lies with the smearing of the discontinuity caused by the advection, which introduces a nonequilibrium state into the calculation making to appear numerical fronts that propagate at non-physical speeds [23]. The model problem is a scalar linear advection equation with a nonlinear reaction source term, which can be stiff. The parameter $\beta$ controls the stiffness character of the model. For $\beta>0$, the associated ODE has stable equilibria at $u=0$ and $u=1$, and an unstable equilibrium precisely at $u=\mu$.

We also report in Figure 6 a preliminary approximate numerical solution given by scheme [28] proposed in
[21], where it is treated a balance law system problem, in which it is computed solutions for $\mathrm{h}$ (height of the free surface on the left) and for $\mathrm{v}$ (averaged horizontal velocity right). The problem considered is a $2 \times 2$ nonlinear system of balance laws $h_{t}+(h v)_{x}=$ $0 ;(h v)_{t}+\left(v^{2} h+\left(\frac{1}{2}\right) h^{2}\right)_{x}=h-C\left(\frac{1-h}{\tan (\theta)}\right) v^{2}$

where the friction coefficient $\mathrm{C}$ is taken to be 0.1 , with the inclination angle $\theta=\frac{\pi}{6}$. The initial velocity is taken to be $v_{0}=1.699$, while the initial height of the free surface consists of a triangular perturbation of the uniform flow level, $h_{0}(x)=x+1.5,-0.5 \leq x \leq 0$, $h_{0}(x)=-x+1.5,0 \leq x \leq 0.5$ and 1 elsewhere, in which with no friction $(\mathrm{C}=0)$, two symmetrical waves will arise from the announced initial data. On the other hand, the introduction of friction not only slow down the velocity of these waves, but also changes their shape. 


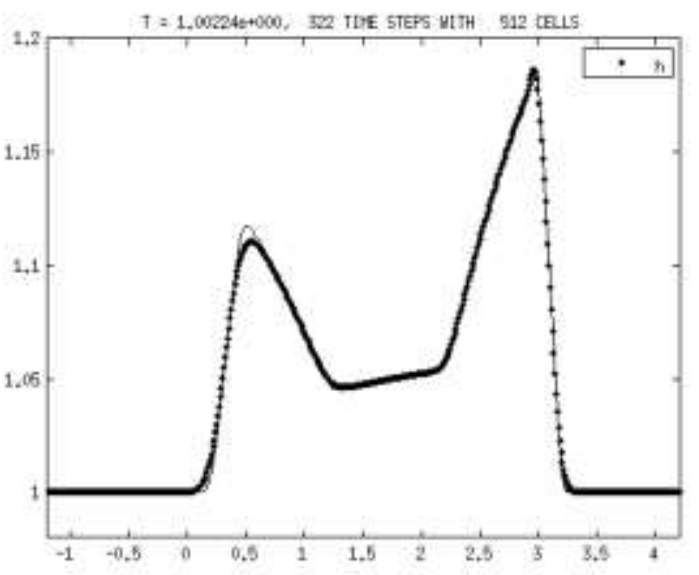

Figure 6. $2 \times 2$ nonlinear system of balance laws with friction.

In order to add one more test problem, see Figure 7, we will use the two-dimensional Lagrangian-Eulerian scheme (9) for the two-dimensional Burgers' equation proposed in [8]. We consider the following twodimensional initial value problem for associated to the inviscid Burgers' equation

$$
\frac{\partial u}{\partial t}+\frac{\partial}{\partial x}\left(\frac{u^{2}}{2}\right)+\frac{\partial}{\partial y}\left(\frac{u^{2}}{2}\right)=0
$$

with $(x, y, t) \in[0,1] \times[0,1] \times[0,0.5]$, and initial condition

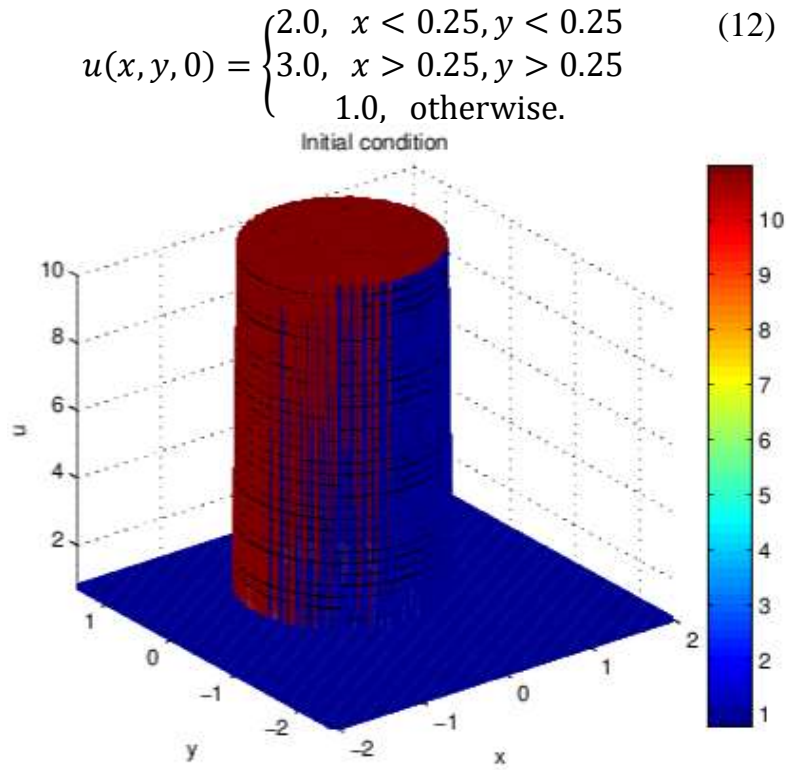

Figure 7. Initial condition.

As before, our numerical solutions are in very good agreement as reported in [8], with a central scheme with a non-structure computational mesh grid. In addition, we

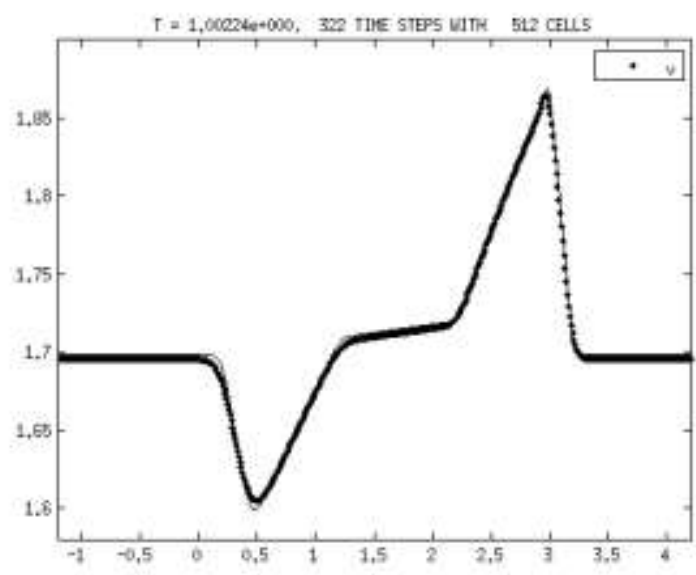

also show a mesh refinement study in order to address the observed numerical order of convergence rate in discrete $L^{p}, p=1,2, \infty$-spaces, (see Tables 4-6).

Table 4. Errors between the numerical approximations $(U)$ and exact solutions $(u)$ in $l_{h}^{1}$ for problem $u_{t}+u_{x}+u_{y}=0$ with initial $u(x, y, 0)=\sin (\pi(x+y))$.

\begin{tabular}{|c|c|c|}
\hline Cells & $\mathrm{h}$ & $\|u-U\|_{l_{h}^{1}}$ \\
\hline $64 \times 64$ & 0.016 & $5.156 \times 10^{-2}$ \\
\hline $128 \times 128$ & 0.007 & $2.046 \times 10^{-2}$ \\
\hline $256 \times 256$ & 0.004 & $1.309 \times 10^{-2}$ \\
\hline $512 \times 512$ & 0.002 & $6.090 \times 10^{-3}$ \\
\hline LSF $E(h)$ & & $2.911 h^{0.989}$ \\
\hline
\end{tabular}

Source: Author development.

Table 5. Errors between the numerical approximations $(U)$ and exact solutions $(u)$ in $l_{h}^{2}$ for problem $u_{t}+u_{x}+u_{y}=0$ with initial $u(x, y, 0)=\sin (\pi(x+y))$.

\begin{tabular}{|c|c|c|}
\hline Cells & $\mathrm{h}$ & $\|u-U\|_{l_{h}^{1}}$ \\
\hline $64 \times 64$ & 0.016 & $5.573 \times 10^{-2}$ \\
\hline $128 \times 128$ & 0.007 & $2.493 \times 10^{-2}$ \\
\hline $256 \times 256$ & 0.004 & $1.467 \times 10^{-2}$ \\
\hline $512 \times 512$ & 0.002 & $6.034 \times 10^{-3}$ \\
\hline LSF $E(h)$ & & $3.051 h^{0.972}$ \\
\hline
\end{tabular}

Source: Author development.

Table 6. Errors between the numerical approximations $(U)$ and exact solutions $(u)$ in $l_{h}^{\infty}$ for problem $u_{t}+u_{x}+u_{y}=0$ with initial $u(x, y, 0)=\sin (\pi(x+y))$.

\begin{tabular}{|c|c|c|}
\hline Cells & $\mathrm{h}$ & $\|u-U\|_{l_{h}^{1}}$ \\
\hline $64 \times 64$ & 0.016 & $1.339 \times 10^{-1}$ \\
\hline $128 \times 128$ & 0.007 & $6.509 \times 10^{-2}$ \\
\hline $256 \times 256$ & 0.004 & $3.761 \times 10^{-2}$ \\
\hline $512 \times 512$ & 0.002 & $1.835 \times 10^{-2}$ \\
\hline LSF $E(h)$ & & $6.534 h^{0.939}$ \\
\hline
\end{tabular}

Source: Author development. 

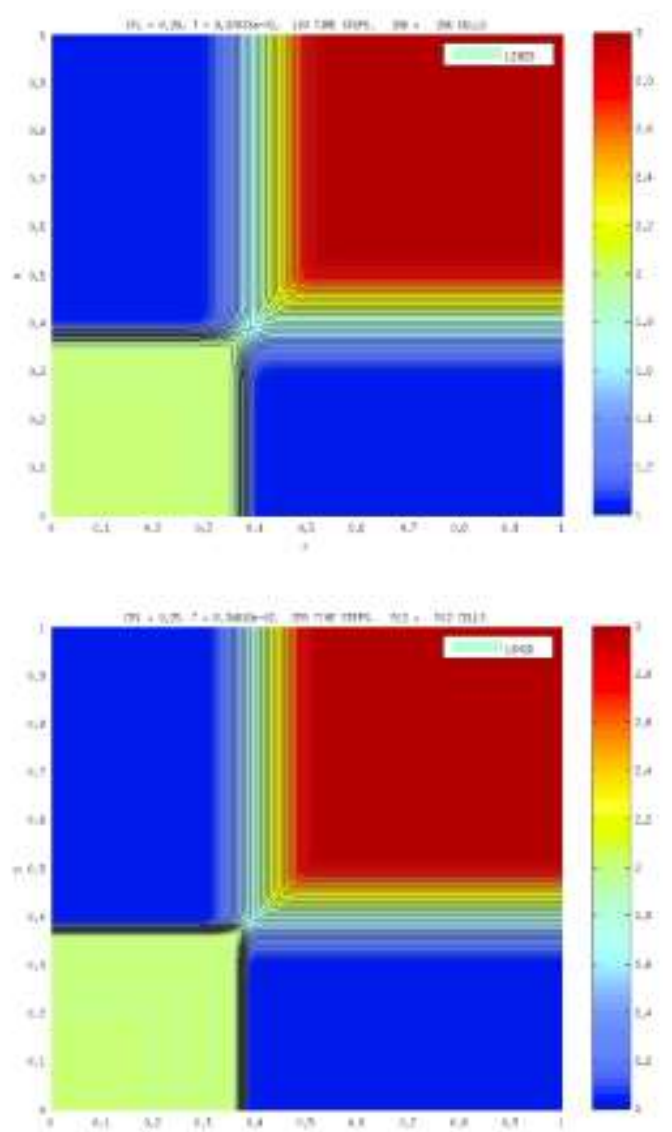
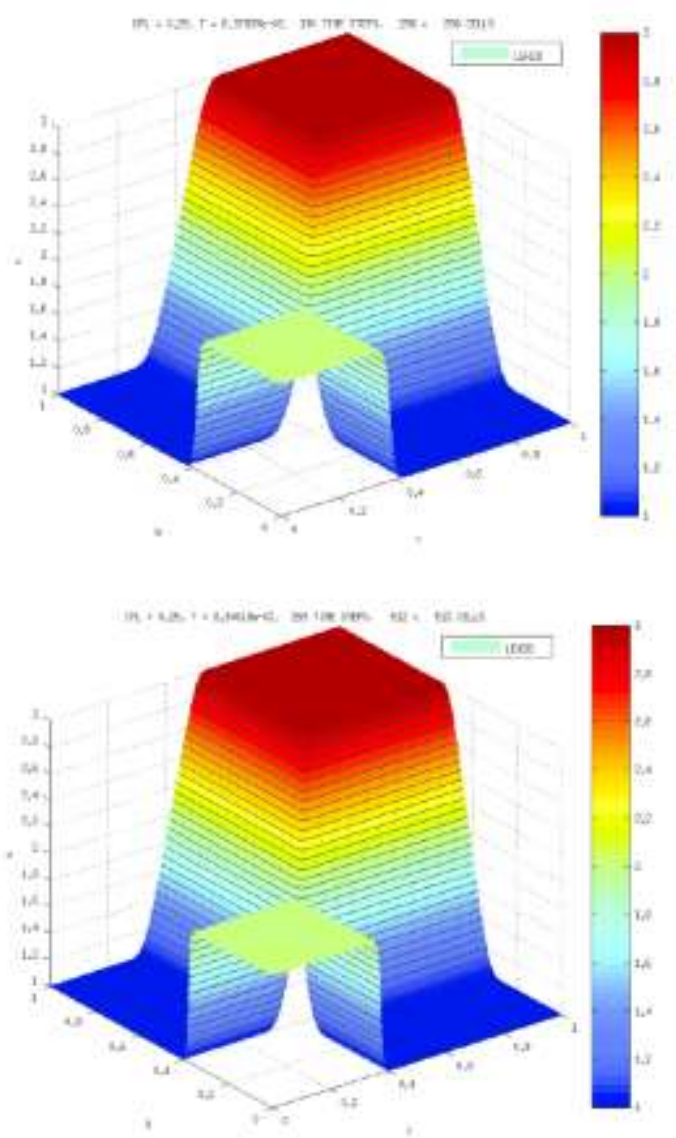

Figure 8. Christov \& Popov (JPC), two-dimensional Burgers' equation [8]. Approximations with the new approach (9)-(10).

\section{CONCLUDING REMARKS}

The new locally conservative Lagrangian method proposed in [28] seems to be promising for the approximation of nonlinear problems in 1D and 2D for hyperbolic conservation laws and balance laws, scalar and system (in this work we discuss an original extension for two-dimensional problems). To the best of our knowledge we have introduced a new approach for construction of a new family of approximate solutions for multidimensional hyperbolic conservation laws (as well as for balance law problems) thanks to an appropriate reformulation of the original differential governing equation by means of an equivalent system of balance laws. Thus, in order to establish such connection between multidimensional hyperbolic conservation laws and system of balance laws, it will be necessary to revisit the Lagrangian-Eulerian framework in order to perform a rigorous numerical analysis as such given in [28] for discrete $L^{p}, p=1,2, \infty$-spaces, but keeping good mathematical properties such as the asymptotic preserving and the well-balancedness of the scheme, as in modern numerical procedures to this end (see, e.g.,
[16]) for hyperbolic conservation laws and balance laws linked to (quasi-)steady solutions.

In [28], we also derived the modified equation associated to the Lagrangian-Eulerian scheme and described the dispersive dissipative relation to explain the onedimensional numerical experiments results. We were able to write the Lagrangian-Eulerian scheme in conservative form for nonlinear hyperbolic conservation laws as well as we construct a Lipschitz continuous consistency condition to the Lagrangian-Eulerian numerical flux function. The Harten theory [18, 19], the Majda and Crandall theory [9] and the ideas in Smoller's book [29] were used to prove the convergence of the approximate solutions, obtained using our LagrangianEulerian scheme for entropic solution of non-linear scalar hyperbolic conservation law in both numerical methods. Furthermore, numerical experiments for hyperbolic conservation laws with convex and non-convex flux functions were also present to illustrate the qualities of the new scheme. In particular, it was discussed the stability and convergence issues of the LagrangianEulerian scheme for linear and nonlinear balance law problems. 


\section{ACKNOWLEDGEMENT}

Eduardo Abreu thanks in part by FAPESP 2016/23374-1 and CNPq Universal 445758/2014-7. John Perez thanks, CAPES-IMECC/UNICAMP and ITM-Institucion Universitaria. Arthur Santo thanks, CAPES-IMECC/ UNICAMP and CNPq-IMECC/UNICAMP

\section{REFERENCES}

[1] E. Abreu and W. Lambert and J. Perez and A. Santo, A new finite volume approach for transport models and related applications with balancing source terms, Mathematics and Computers in Simulation, 137(2017) 228.

[2] J. Aquino, F. Pereira, H. P. Amaral Souto, A. S. Francisco. A forward tracking scheme for solving radionuclide advective problems in unsaturated porous media, Int. J. of Nuclear Energy Science and Technology, 3(2) (2007), 196-205.

[3] J. Aquino et al.A Lagrangian strategy for the numerical simulation of radionuclide transport problems, Progress in Nuclear Energy, 52(3), 282-291, 2010.

[4] A. Bressan and W. Shen, Optimality conditions for solutions to hyperbolic balance laws, Contemporary Mathematics, 426, 129-152, 2007.

[5] S.-C. Chang, A critical analysis of the modified equation technique of Warming and Hyett, Journal of Computational Physics, 86(1), 107-126, 1990.

[6] A. Chalabi, On convergence of numerical schemes for hyperbolic conservation laws with stiff source terms, Mathematics of Computation of the American Mathematical Society, 66(218), 527-545, 1997.

[7] G.-Q. Chen C. D. Levermore and T.-P. Liu, Hyperbolic conservation laws with stiff relaxation terms and entropy, Communications on Pure and Applied Mathematics, 47(6),787-830, 1994.

[8] I. Christov and B. Popov, New non-oscillatory central schemes on unstructured triangulations for hyperbolic systems of conservation laws, Journal of Computational Physics 227 (2008) 5736-5757.

[9] M. G. Crandall and A. Majda. Monotone difference approximations for scalar conservation laws. Mathematics of Computation, 34(149):1-21, 1980.

[10] C. M. Dafermos Hyperbolic conservation laws in continuous physics, 2nd edition SpringerVerlag,227(11), pags 1-626, 2005.
[11] R. Donat and I. Higueras and A. Martinez-Gavara, On stability issues for IMEX schemes applied to 1D scalar hyperbolic equations with stiff reaction terms, Mathematics of Computation, 80(276), 2097-2126, 2011.

[12] J. Douglas, Jr, Finite difference methods for twophase incompressible flow in porous media, SIAM Journal on Numerical Analysis, 20(4), 681-696, 1983.

[13] J. Douglas, Jr and C.-S. Huang. A locally conservative eulerian-lagrangian finite difference method for a parabolic equation. BIT Numerical Mathematics, 41(3):480-489, 2001.

[14] J. Douglas, F. Pereira and L. M. Yeh. A locally conservative Eulerian-Lagrangian numerical method and its application to nonlinear transport in porous media, Computational Geosciences, 4 (2000), 1-40.

[15] J. Douglas, Jr and T. F. Russell. Numerical methods for convection-dominated diffusion problems based on combining the method of characteristics with finite element or finite difference procedures. SIAM Journal on Numerical Analysis, 19(5):871-885, 1982.

[16] L. Gosse Computing Qualitatively Correct Approximations of Balance Laws Exponential-Fit, WellBalanced and Asymptotic-Preserving SIMAI Springer Series (2013) vol 2.

[17] D. F. Griffiths and J. M. Sanz-Serna, On the scope of the method of modified equations, SIAM Journal on Scientific and Statistical Computing, 7(3), 994-1008, 1986.

[18] A. Harten. High resolution schemes for hyperbolic conservation laws. Journal of computational physics, 49(3):357-393, 1983.

[19] A. Harten, J. M. Hyman, P. D. Lax, and B. Keyfitz. On finite-difference approximations and entropy conditions for shocks. Communications on pure and applied mathematics, 29(3):297-322, 1976.

[20] C. S. Huang, T. Arbogast and J. Qiu. An EulerianLagrangian WENO finite volume scheme for advection problems, J. Comp. Phys., 231(11) (2012), 4028-4052.

[21] J. O. Langseth, A. Tveito, and R. Winther, On the Convergence of Operator Spliting Applied to Conservation Laws with Source Terms SIAM J. Numer. Anal. 33(3) (1996) 843-863. 
[22] P. G. LeFloch, Hyperbolic Systems of Conservation Laws: The theory of classical and nonclassical shock waves, Springer Science and Business Media, 2002.

[23] R. J. Leveque and H. C. Yee, A study of numerical methods for hyperbolic conservation laws with stiff source terms, Journal of Computational, Physics 86(1) (1990) 187-210.

[24] T. P. Liu, Linear and nonlinear large-time behavior of solutions of general systems of hyperbolic conservation laws, Communications on Pure and Applied Mathematics, 30(6), 767-796, 1977.

[25] T. P. Liu, Quasilinear hyperbolic systems, Communications in Mathematical Physics, 68(2), 141172, 1979.

[26] S. Mancuso, F. Pereira, and G. de Souza. Um novo m'etodo Euleriano-Lagrangeano para aproximação de leis de conservação doi: 10.5540/tema. 2007.08. 02.0277. Trends in Applied and Computational Mathematics, 8(2):277-286, 2007

[27] L. Pareschi Central differencing based numerical schemes for hyperbolic conservation laws with relaxation terms, SIAM Journal on Numerical Analysis, 39(4), 1395-1417, 2001.

[28] J. A. Perez, Lagrangian-Eulerian approximation methods for balance laws and hyperbolic conservation laws, Institute of Mathematics, Statistics and Scientific Computing, University of Campinas, PhD. Thesis. 2015. [29] J. Smoller. Shock waves and reaction-diffusion equations, volume 258. Springer Science and Business Media, 1994.

[30] H. Wang, D. Liang, R. E. Ewing, S.-L. Lyons and G. Qin, An ELLAM approximation for highly compressible multicomponent flows in porous media, Computational Geosciences, 6(3-4),227-251, 2002.

[31] R. F. Warming and B. J. Hyett, The modified equation approach to the stability and accuracy analysis of finite-difference methods, Journal of computational physics, 14(2), 159-179, 1974.

[32] D. Fuentes-Diaz, P. Díaz-Guerrero and R. Sánchez, "Cálculo del flujo difusivo en dominios complejos mediante el método de volúmenes finitos," Rev. UIS Ing., vol. 11, no. 1, pp.45-54, 2012. 